\title{
Glucocorticoid receptors are downregulated in hepatic T lymphocytes in rats with experimental cholangitis
}

\author{
K Tjandra, T Le, M G Swain
}

Gut 2003;52:1363-1370

\begin{abstract}
Background and aims: Primary sclerosing cholangitis is a Th 1 cytokine driven disease with a poor clinical responsiveness to glucocorticoid therapy. We have previously documented elevated circulating glucocorticoid levels in cholestatic rats and in addition have noted increased hepatic expression of the Th 1 cytokine interferon $\gamma$ (IFN- $\gamma$ ) in a rat model of cholangitis. Therefore, we examined the relationship between circulating glucocorticoid levels, hepatic IFN- $\gamma$ expression, and hepatic T cell glucocorticoid receptor (GR) expression in a rat model of cholangitis to provide insight into the possible mechanism underlying hepatic T cell glucocorticoid resistance in cholangitic diseases. Methods: Cholangitis was induced in male Sprague-Dawley rats by oral administration of low dose $\alpha$-naphthylisothiocyanate (ANIT). On day 14, ANIT fed and control rats were sacrificed, serum collected, and hepatic, splenic, and peripheral blood T lymphocytes isolated for GR expression, as determined by reverse transcription-polymerase chain reaction and western blotting. Results: Circulating glucocorticoid levels were markedly elevated in ANIT fed rats. Hepatic T lymphocyte GR mRNA and protein levels were significantly reduced in ANIT treated rats compared with controls. In contrast, GR mRNA and protein expression in splenic and circulating T lymphocytes was similar in both groups. Furthermore, reduced hepatic T cell GR expression in ANIT fed rats was associated with reduced hepatic $C D 4^{+} \mathrm{T}$ cell sensitivity to dexamethasone inhibitory effects (that is, inhibition of interleukin 2 receptor expression).

Conclusion: We conclude that hepatic T lymphocyte resistance to elevated endogenous glucocorticoid levels in rats with experimental cholangitis appears, in part, to be mediated by decreased GR expression.
\end{abstract}

See end of article for authors' affiliations

Correspondence to: DrM G Swain, Gastrointestinal Research Group, Health Sciences Center, 3330 Hospital Drive NW, Calgary, Alberta, Canada T2N

1N4; swain@ucalgary.ca 15 April 2003

$\mathrm{P}$ rimary sclerosing cholangitis (PSC) is a chronic cholestatic liver disease characterised by an obliterative inflammatory fibrosis of the biliary tract, which slowly progresses to cirrhosis and liver failure..$^{1-3}$ The cause of PSC is unknown and consequently appropriate therapy for this disease remains a tremendous challenge for clinicians. PSC, a presumed autoimmune disease, has been associated with the development of a predominant hepatic Thl cytokine profile. Recently, we demonstrated that experimental cholangitis in rats is associated with the progressive development of a Thl hepatic cytokine profile, specifically through overproduction of interferon $\gamma$ (IFN- $\gamma$ ) by hepatic CD4 positive lymphocytes.'

Glucocorticoids have been shown to inhibit the production of Thl cytokines. ${ }^{6}$ In contrast, a major adrenal hormone dehydroepiandrosterone (DHEA) has been shown to enhance Thl cytokine production, such as interleukin (IL) -2 and IFN- $\gamma^{7}{ }^{7}$ Therefore, the endogenous steroid milieu may be important in controlling the cytokine microenvironment during an inflammatory process such as chronic cholangitis. Moreover, we have previously demonstrated increased circulating glucocorticoid levels in rats with chronic cholestasis due to bile duct ligation. ${ }^{9}$

Although corticosteroids are generally useful for the treatment of inflammatory diseases (for example, inflammatory bowel disease (IBD) and asthma), some patients are nonresponsive to the immunosuppressive effects of these drugs and have been termed "steroid resistant". The mechanism underlying this steroid resistance is not fully understood, although there is evidence to suggest that glucocorticoid receptor (GR) defects may contribute to the observed phenomena. ${ }^{10-13}$ Interestingly, in chronic cholestatic liver diseases such as PSC and primary biliary cirrhosis (PBC), steroid treatment alone or in combination with other drugs does not appear to be effective in treating these presumed

immune mediated diseases. ${ }^{14-16}$ It is not clear however whether the poor response to steroid treatment in these patients is due specifically to glucocorticoid resistance.

The objectives of this study were to determine whether circulating endogenous glucocorticoid levels are increased in rats with experimental cholangitis, and if they are, to determine whether the development of a hepatic Thl cytokine profile is due to changes in hepatic $\mathrm{T}$ lymphocytes in the setting of cholangitis which may render them less responsive to glucocorticoids.

\section{MATERIALS AND METHODS}

\section{Animal model of chronic cholangitis}

Experimental cholangitis was induced in male SpragueDawley rats (200-250 g; Charles River, St Constant, Quebec, Canada) by chronic oral administration of low dose $\alpha$ - naphthylisothiocyanate (ANIT $1 \mathrm{mg} / \mathrm{kg}$ powdered rat chow; Sigma Chemicals, St Louis, Missouri, USA) as previously described. Both control and ANIT treated rats were kept in a light controlled room (12 hour light/12 hour dark cycles) and treated in accordance with the Canadian Council of Animal Care guidelines. Rats were handled twice daily to avoid

Abbreviations: PSC, primary sclerosing cholangitis; IL, interleukin IL-2R, IL-2 receptor; IFN, interferon; DHEA, dehydroepiandrosterone; $\mathrm{IBD}$, inflammatory bowel disease; GR, glucocorticoid receptor; $\mathrm{PBC}$, primary biliary cirrhosis; ANIT, $\alpha$-naphthylisothiocyanate; PBS, phosphate buffered saline; FITC, fluorescein isothiocyanate; PE phycoerythrin; RT-PCR, reverse transcription-polymerase chain reaction TNF, tumour necrosis factor; FACS, fluorescence activated cell sorter; ConA, concanavalin A; NK, natural killer; GAPDH, glyceraldehyde-3. phosphate dehydrogenase; PBMC, peripheral blood mononuclear cells. 

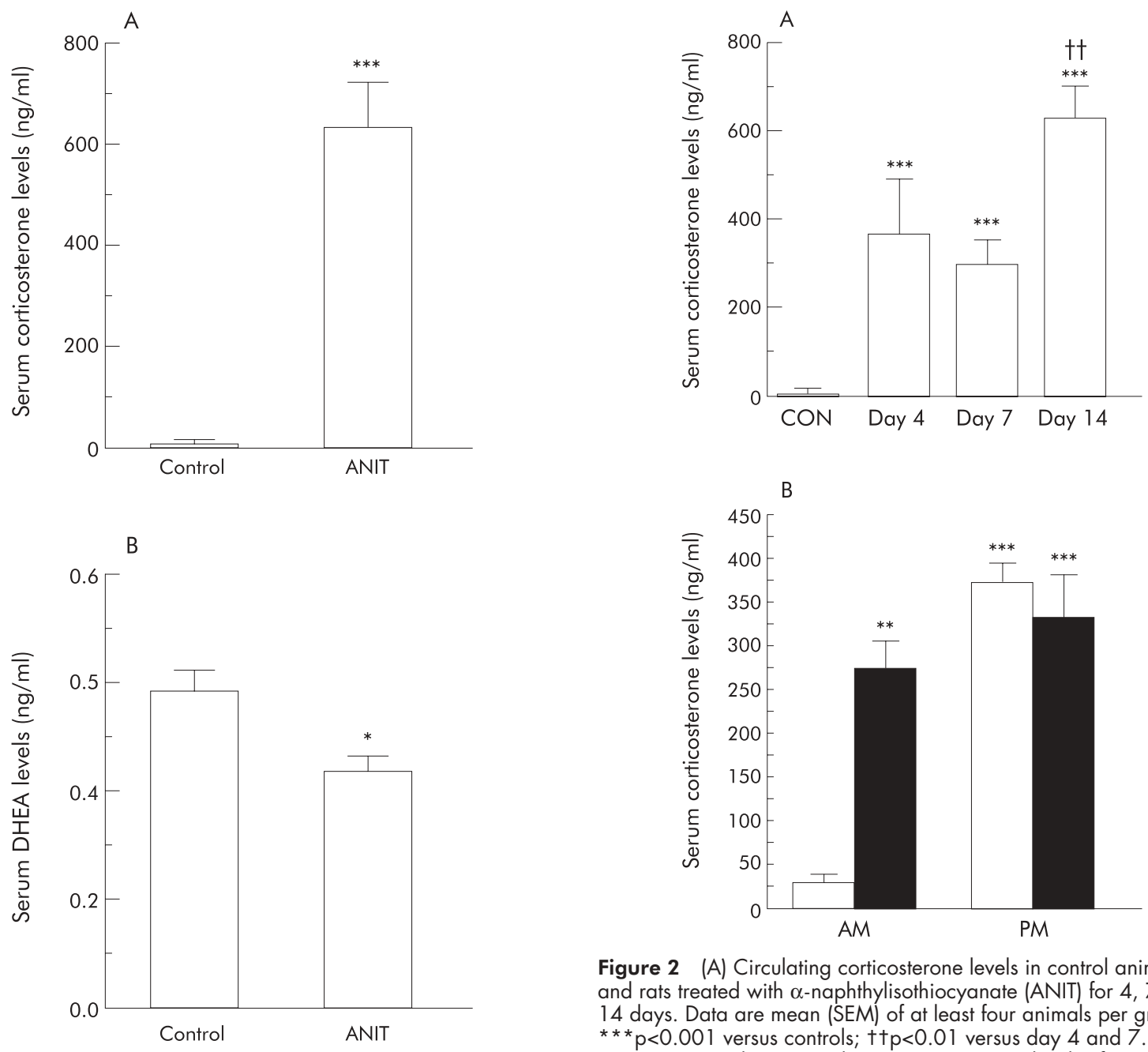

Figure 2 (A) Circulating corticosterone levels in control animals and rats treated with $\alpha$-naphthylisothiocyanate (ANIT) for 4, 7, and 14 days. Data are mean (SEM) of at least four animals per group. $* * * p<0.001$ versus controls; $\uparrow+p<0.01$ versus day 4 and 7 .

(B) Morning and evening plasma corticosterone levels of control and ANIT fed rats for 14 days. Data are mean (SEM) of six animals per control group, and at least 13 rats per ANIT group. ${ }^{*} p<0.01$,

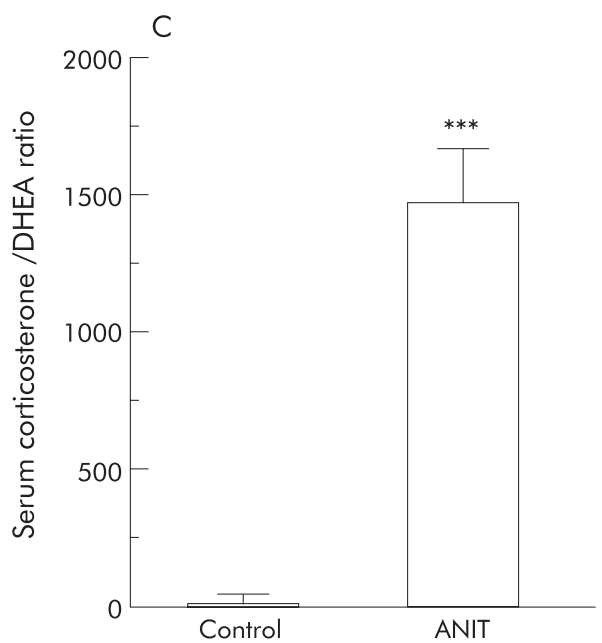

$* * * p<0.001$ versus control AM levels.

expression. Experiments were also performed to determine serum corticosterone kinetics: (i) serum corticosterone levels were also measured four and seven days following ANIT administration and (ii) corticosterone diurnal variations were examined in control rats and rats treated with ANIT for 14 days according to Sarlis and colleagues. ${ }^{17}$ Rat truncal blood was collected in the morning (AM: between 8 and $9 \mathrm{am}$ ) and in the evening (PM: between 7 and 8 pm).

\section{Serum corticosterone and DHEA levels}

Serum corticosterone (the major adrenal glucocorticoid found in rodents) and DHEA levels of control and ANIT treated rats were determined using commercially available radioimmunoassays (ICN Biomedicals, Inc. Costa Mesa, California, USA, and Diagnostic System Laboratories, Inc., Webster, Texas, USA, respectively).

\section{Hepatic cytokine levels}

Livers of control and ANIT treated rats were perfused with ice cold phosphate buffered saline (PBS; pH 7.4) and extracted for cytokine protein expression as previously described. ${ }^{5}$ Hepatic Th1 (IFN- $\gamma$ ) and Th2 (IL-4) cytokines were quantitated by commercially available ELISA kits (Medicorp, Montreal, Quebec, Canada).

handling stress and maintain low baseline serum corticosteroid levels. Animals were sacrificed 14 days following ANIT treatment, truncal blood was collected for serum steroid levels, and the liver perfused and removed for cytokine protein

\section{Hepatic T lymphocyte isolation}

Hepatic lymphocytes were isolated as previously described. ${ }^{5}$ Liver derived lymphocytes were used for determination of GR 
mRNA/protein expression and cellular surface markers. T lymphocytes from the spleen and peripheral blood of control and ANIT treated rats were isolated with slightly modified protocols. Spleens were excised, minced, and homogenised in PBS. The spleen homogenate was then filtered through a 30 micron nylon mesh (Small Parts Inc.). Cell suspensions were layered on Lympholyte Rat (Cedarlane, Hornby, Ontario, Canada) and centrifuged at $1400 \mathrm{~g}$ for 30 minutes at room temperature. Peripheral blood was collected in sterile tubes containing anticoagulant $(0.14 \mathrm{~mol} / \mathrm{l}$ citric acid, $0.2 \mathrm{~mol} / \mathrm{l}$ sodium citrate, and $0.22 \mathrm{~mol} / \mathrm{l}$ dextrose) and centrifuged at $1400 \mathrm{~g}$ for 10 minutes. The pellets were resuspended in PBS to twice the original volume collected. Cell suspension was then layered on Lympholyte Mammals (Cedarlane) and centrifuged at $800 \mathrm{~g}$ for 30 minutes at room temperature. Splenic and peripheral blood $\mathrm{T}$ lymphocytes were collected from the interface layer, washed, and counted. Purified T lymphocytes were either used immediately or stored at $-70^{\circ} \mathrm{C}$.

\section{Flow cytometry}

To determine the cellular phenotypes and distribution of isolated liver derived lymphocytes in control and ANIT treated animals, we stained these cells for commonly expressed specific cell surface markers and analysed them by flow cytometry. Briefly, isolated hepatic lymphocytes $\left(10^{6}\right.$ cells $)$ were incubated for 30 minutes at room temperature with $10 \mu \mathrm{l}$ of fluorescein isothiocyanate (FITC) conjugated mouse antirat CD4 (clone W3/25; Serotec Inc., Raleigh, New Jersey, USA), mouse antirat CD8 (clone MRC OX-8; Serotec), mouse antirat monocytes/macrophages (clone EDl; Serotec), or mouse antirat $\mathrm{B}$ cell leucocyte common antigen (clone MRC OX-33; Cedarlane). Hepatic natural killer (NK) cells were also determined using phycoerythrin (PE) conjugated mouse antirat NKR-P1A cell surface marker (clone 10/78; Pharmingen, Mississauga, Ontario, Canada), which were labelled together with either FITC conjugated anti-CD4 or anti-CD8 monoclonal antibodies (see above). Mouse FITC (Serotec) and PE (Pharmingen) conjugated IgGl negative isotype controls were also included in the analysis. Cells were washed twice in PBS following incubation, and resuspended in $0.2 \mathrm{ml}$ of $1 \%$ formalin in PBS. Fixed cells were stored overnight at $4^{\circ} \mathrm{C}$ and analysed the next day by flow cytometry (Becton-Dickinson, Mountain View, California, USA). Cells were counted using an electronic gate set on the lymphocyte cluster on the forward and side scatter plot and analysed using CellQuest software (Becton- Dickinson).

\section{Semiquantitative RT-PCR}

RNA was extracted from purified T lymphocytes isolated from various sources (that is, liver, spleen, and peripheral blood) in Trizol (Molecular Research Center, Inc., Cincinnati, Ohio, USA) according to the manufacturer's protocol. Briefly, isolated cells were sonicated in $1 \mathrm{ml}$ of ice cold Trizol for 2-3 minutes (Fisher sonic dismembrator model 300; Farmingdale, New York, USA) and RNA extracted by adding $0.2 \mathrm{ml}$ chloroform. Following centrifugation at $12000 \mathrm{~g}$ for 15 minutes at $4^{\circ} \mathrm{C}$, the aqueous phase was transferred to an Eppendorf tube and precipitated in $0.5 \mathrm{ml}$ isopropanol. Further centrifugation at $12000 \mathrm{~g}$ for 15 minutes at $4^{\circ} \mathrm{C}$ resulted in an RNA pellet that was washed with $1 \mathrm{ml}$ of $75 \%$ ethanol and centrifuged at $7500 \mathrm{~g}$ for five minutes. The pellet was air dried and dissolved in diethyl pyrocarbonate treated water (Research Genetics, Burlington, Ontario, Canada) and stored at $-70^{\circ} \mathrm{C}$. The final RNA concentration was determined spectrophotometrically using a Gene Quant spectrophotometer (Pharmacia, Piscataway, New Jersey, USA). All reagents were from Sigma, unless otherwise indicated.

Steady state T lymphocyte GR mRNA levels were determined using the "primer dropping" method previously described. ${ }^{518}$ Reverse transcription-polymerase chain reaction (RT- PCR) was performed in an Amplitron I Thermal Cycler

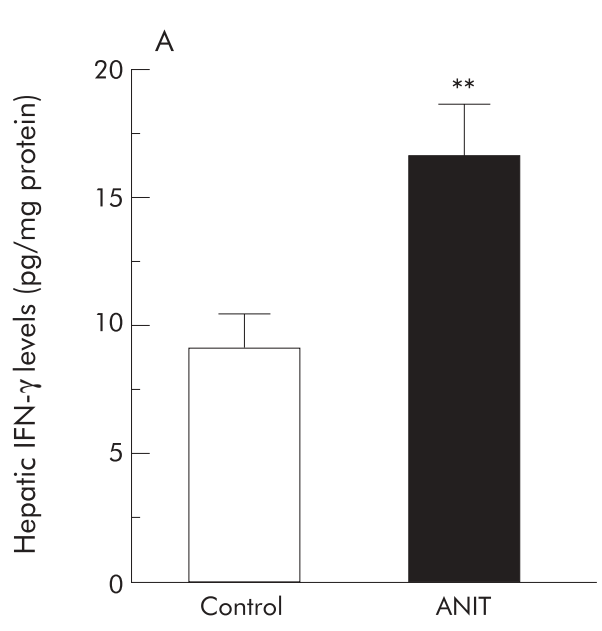

(Barnsted/Thermolyne, Dubuque, Iowa, USA). Briefly, complementary DNA (cDNA) was generated by an RT reaction by preincubating $2 \mu \mathrm{g}$ of sample RNA with the appropriate RT reaction mixture at $21^{\circ} \mathrm{C}$ for 10 minutes, $42^{\circ} \mathrm{C}$ for 50 minutes, and $95^{\circ} \mathrm{C}$ for five minutes to terminate the reaction. Multiple 

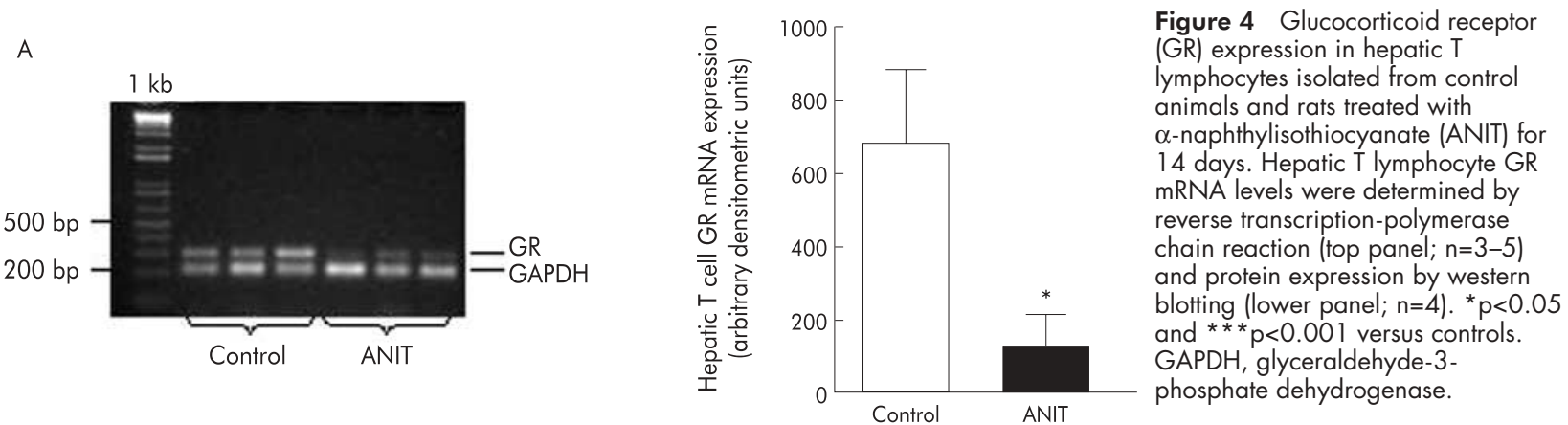

B

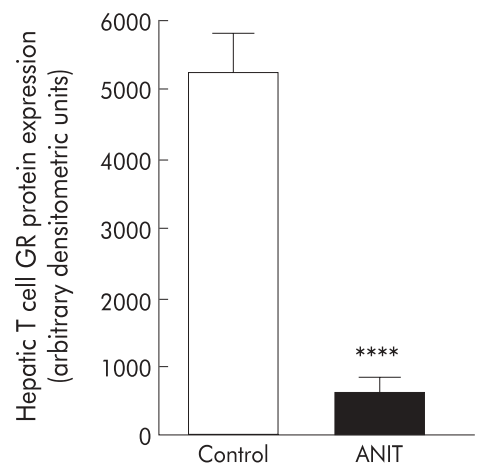

PCR reactions were performed using $2 \mu \mathrm{l}$ of the newly synthesised CDNA as a template and amplified in a $50 \mu \mathrm{l} \mathrm{PCR} \mathrm{reaction}$ mixture containing $1 \times \mathrm{PCR}$ buffer, $80 \mu \mathrm{M}$ of each deoxynucleotide triphosphates (dATP, dGTP, dCTP, and dTTP), and 20 pmol each of rat GR primers (307 bp; Biognostik, Germany). Each PCR cycle consisted of a heat denaturation step at $94^{\circ} \mathrm{C}$ for one

minute, a primer annealing step at $60^{\circ} \mathrm{C}$ for 30 seconds, and a polymerisation step at $72^{\circ} \mathrm{C}$ for one minute. For proper amplification and to ensure that PCR amplification was in the linear range, the number of PCR cycles was predetermined for each sample (liver, 34 cycles; spleen and peripheral blood, 33 cycles). Two units of Taq DNA Polymerase (Pharmacia) were

A

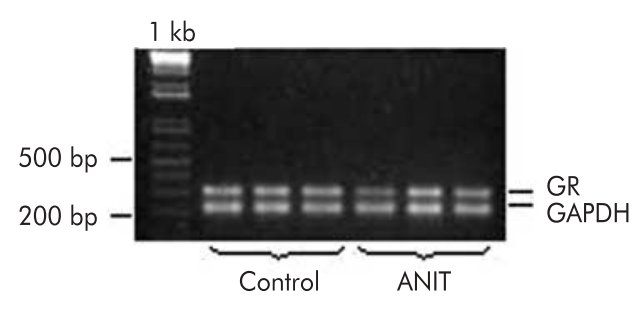

B

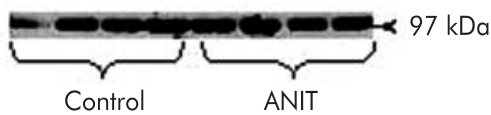

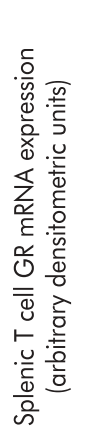

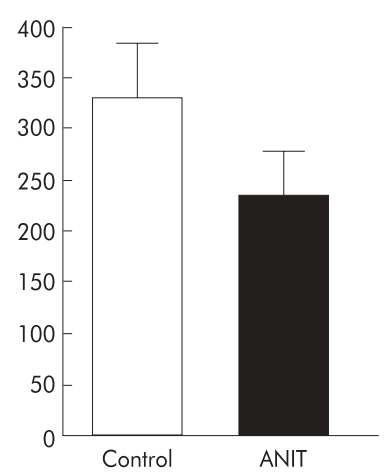

Figure 5 Splenic T lymphocyte glucocorticoid receptor (GR) expression in control and rats receiving $\alpha$-naphthylisothiocyanate (ANIT) for 14 days. Splenic T lymphocyte GR mRNA levels are shown in the top panel $(n=9)$ and protein expression in the lower panel $(n=4)$. Similar splenic T lymphocyte GR mRNA and protein levels were observed between the control rats and those receiving ANIT for 14 days. GAPDH, glyceraldehyde-3 -phosphate dehydrogenase.

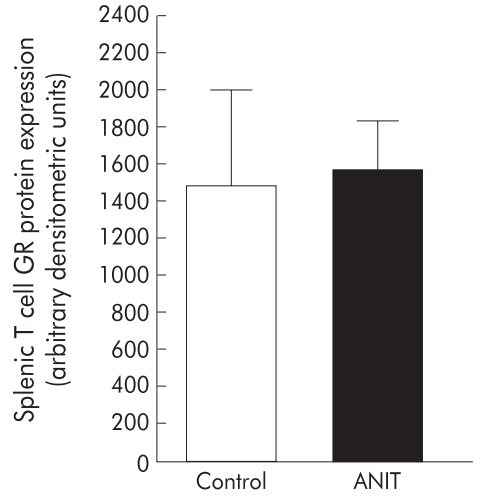


A

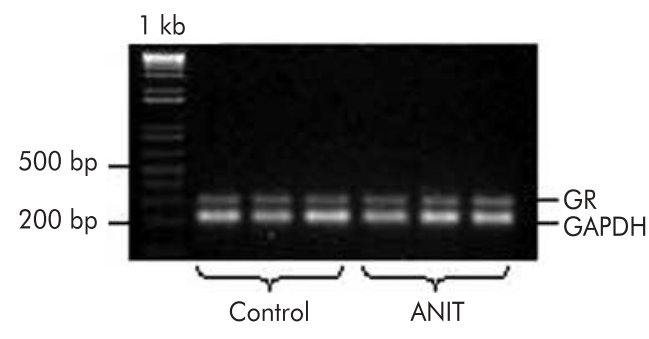

B

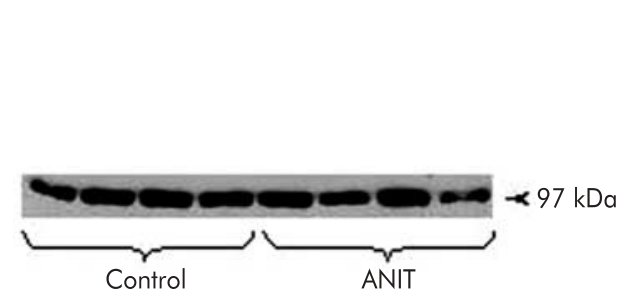

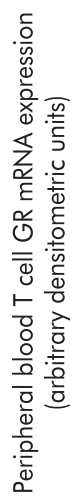

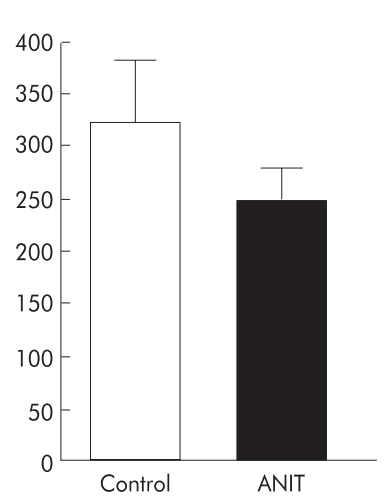

Figure 6 Peripheral blood T

lymphocyte glucocorticoid receptor (GR) expression in control and rats receiving $\alpha$-naphthylisothiocyanate (ANIT) for 14 days. Circulating T lymphocyte GR mRNA levels are shown in the top panel $(n=7-8)$ and protein expression in the lower panel $(n=4)$. Similar GR mRNA and protein levels in peripheral blood $T$ lymphocytes were observed between the control rats and those receiving ANIT for 14 days.

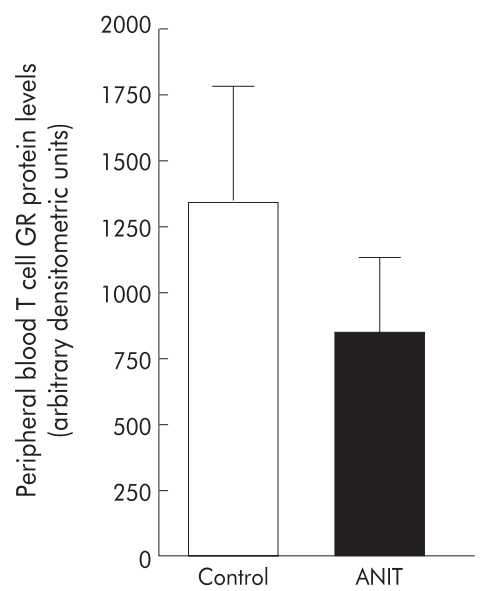

antibody (1/3000; Jackson Immuno Research Laboratories Inc., West Grove, Pennsylvania, USA). Positive immunoreactivity on blots was detected following exposure to a chemiluminescent producing substrate (Pierce, Rockford, Illinois, USA). GR protein band density was quantitated using computer densitometric scanning of the images with Quantity One software (Biorad).

\section{GR sensitivity assay}

To determine whether reduced hepatic T cell GR number in chronic cholangitis is associated with reduced GR sensitivity to glucocorticoid, surface IL-2 receptor (IL-2R) expression (IL-2R expression is a widely used marker of lymphocyte activation $^{21}$ ) on hepatic $\mathrm{CD}^{+}$lymphocytes was determined using flow cytometry, as described previously. ${ }^{22}$ Briefly, liver derived lymphocytes from control and ANIT fed rats were incubated in RPMI 1640 media supplemented with 5\% fetal calf serum, $100 \mathrm{U} / \mathrm{ml}$ penicillin, $100 \mu \mathrm{g} / \mathrm{ml}$ streptomycin (all from Gibco $\mathrm{BRL}$ ), and $2 \mathrm{mM}$ L-glutamine (Sigma). Liver derived lymphocytes $\left(4 \times 10^{6}\right.$ cells $\left./ \mathrm{ml}\right)$ were stimulated in 24 well plates with concanavalin A (ConA $2.5 \mu \mathrm{g} / \mathrm{ml}$; Sigma) for 24 hours at $37^{\circ} \mathrm{C}$ and $5 \% \mathrm{CO}_{2}$. Then, dexamethasone $\left(10^{-7} \mathrm{M}\right.$; Sigma $)$ or vehicle was added to the cell culture and the plates were further incubated for an additional 24 hours. This dose of dexamethasone has previously been shown to inhibit IL-2R expression during antigen induced mouse splenic $\mathrm{T}$ cell activation. ${ }^{23}$ Cells were harvested, washed, and double stained with FITC conjugated mouse antirat CD4 (clone W3/25; Serotec) followed by PE conjugated mouse antirat CD25 (IL-2R) monoclonal antibody (clone MRC OX-9; Serotec). FITC and PE conjugated mouse IgGl negative controls (Serotec) were also included in the analysis.

\section{Statistics}

All data are expressed as mean (SEM). The Student's $t$ test was used to compare differences between the two groups. Multiple ing them with the primary antibody (mouse monoclon a body against rat GR: 1/200; Affinity BioReagents, Inc., Gold Colorado, USA). Membrane blots were then incubated with goat antimouse horseradish peroxidase linked secondary 

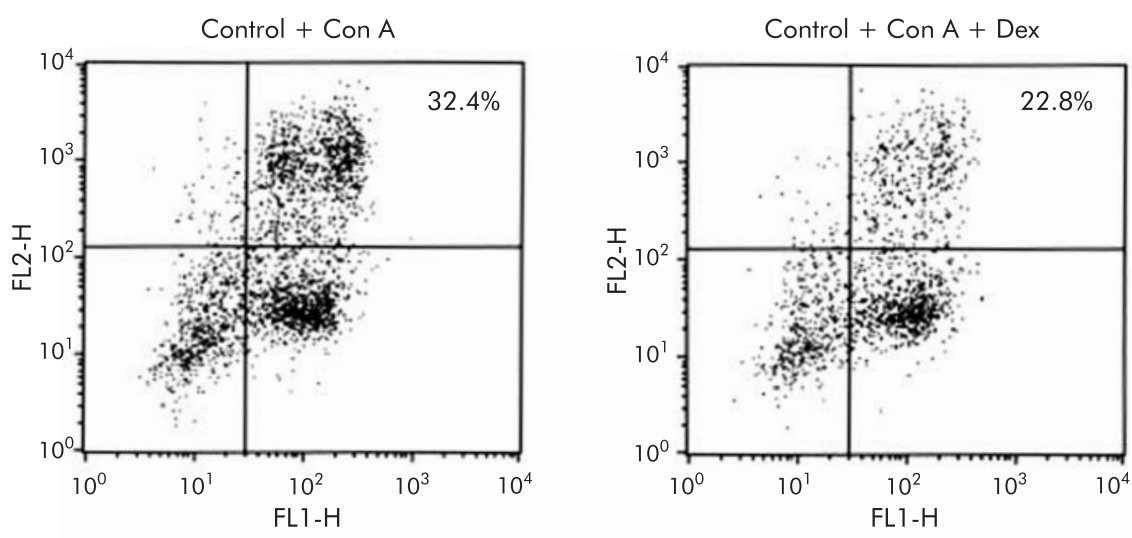

Figure 7 A representative dot plot of surface expression of interleukin 2 receptors (IL- 2R) on $\mathrm{CD} 4^{+}$liver derived lymphocytes of control rats and rats treated with $\alpha$ - naphthylisothiocyanate (ANIT) for 14 days. Freshly isolated hepatic lymphocytes were stimulated with concanavalin A (Con $A$ ) in the presence or absence of $10^{-7} \mathrm{M}$ dexamethasone (Dex). Similar results were obtained in two additional experiments.
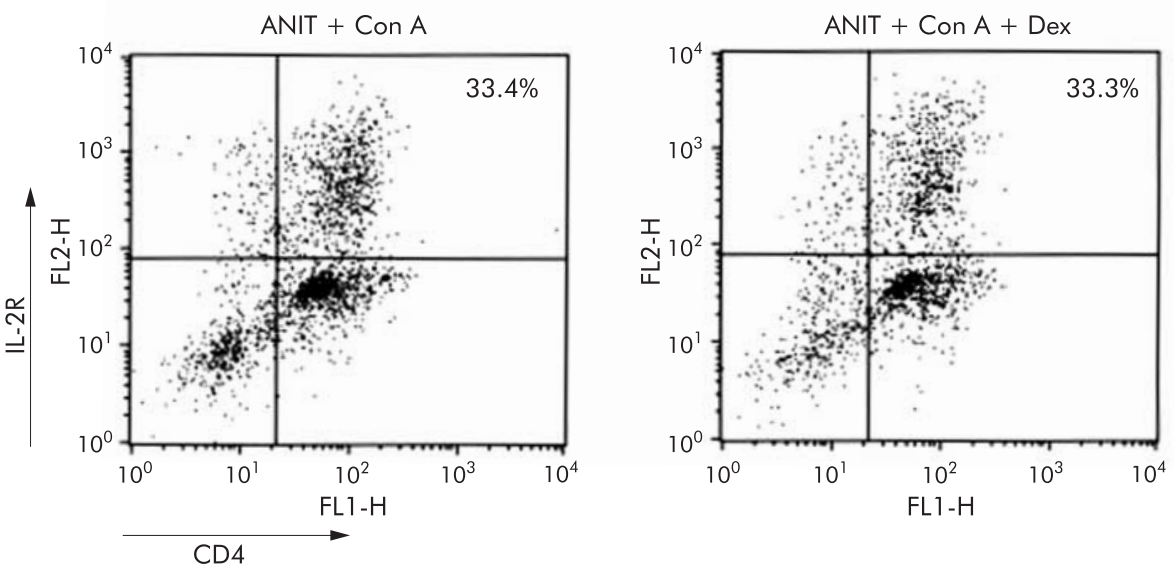

comparisons were performed by one way analysis of variance followed by the Student-Newman-Keul post hoc test. A p value of $<0.05$ was considered significant.

\section{RESULTS}

\section{Serum corticosterone and DHEA levels}

We observed a significant increase in serum corticosterone levels in ANIT treated rats compared with control animals (fig 1A). Serum corticosterone levels were markedly elevated in ANIT fed rats as early as day 4 post ANIT treatment and the elevations were maintained over the remaining 14 days of ANIT treatment (fig 2A). In contrast, serum DHEA levels of rats treated with ANIT were significantly reduced when compared with control levels (fig lB). The serum corticosterone to DHEA ratio was significantly higher in ANIT treated rats than in controls (fig lC). Circulating corticosterone levels in control animals were low in the morning (between 8-9 am) but significantly increased in the evening $(7-8 \mathrm{pm}$ ) (fig $2 \mathrm{~B})$. In contrast, diurnal variation of corticosterone levels were absent in ANIT fed rats; serum corticosterone levels were elevated in the morning and remained high in the evening.

\section{Hepatic cytokine profile}

Hepatic IFN- $\boldsymbol{\gamma}$ levels were significantly elevated in ANIT treated rats compared with controls, but in contrast, no significant difference in hepatic IL-4 levels was noted between control and ANIT treated rats (fig 3A, 3B, respectively). Hepatic IFN- $\gamma / \mathrm{IL}-4$ ratio was significantly higher in ANIT treated rats compared with control animals, giving a predominant Thl hepatic cytokine profile in ANIT treated rats (fig 3C).

\section{Liver derived lymphocytes}

There was no marked difference in the cellular phenotypes and distribution of purified hepatic T lymphocytes obtained from control and ANIT treated rats. However, the total number of hepatic $\mathrm{T}$ lymphocytes extracted from ANIT treated rats was approximately five times higher than from control livers (data not shown). The isolated hepatic lymphocytes mainly consisted of CD4 (control 32 (1)\%; ANIT $32(4) \%$ ) and CD8 positive T lymphocytes (control 74 (3)\%; ANIT 68 (5)\%) with few B cells (control 2 (1)\%; ANIT 1 (2)\%) and monocyte/macrophages (control 3 (1)\%; ANIT 9 (6)\%). Although a large proportion of isolated hepatic lymphocytes were also positive for the NK cell marker (control $62(2) \%$; ANIT $70(4) \%$ ), greater than $90 \%$ of these cells were also CD8 positive. The mean percentage of NK positive liver derived lymphocytes was approximately 10 times higher than that of circulating T lymphocytes (data not shown), consistent with the observations of others. ${ }^{24}$

\section{T lymphocyte GR expression}

Hepatic T lymphocyte GR mRNA expression was significantly reduced in ANIT treated rats compared with control animals (fig 4, top panel). Furthermore, hepatic T lymphocyte GR downregulation in ANIT treated rats was also observed at the protein level (fig 4, bottom panel). To determine whether $\mathrm{T}$ lymphocyte GR downregulation in ANIT treated animals is a generalised phenomenon in response to elevated circulating glucocorticoids, we studied GR mRNA and protein expression of extrahepatic T lymphocytes obtained from the spleen and peripheral blood. In contrast with hepatic T lymphocytes, both splenic and peripheral blood T lymphocytes of control and ANIT treated animals showed similar GR mRNA and protein levels (figs 5,6).

\section{Hepatic $T$ cell IL-2R expression}

In vitro activation of hepatic $\mathrm{T}$ cells with ConA resulted in upregulation of IL-2R expression in both control and ANIT fed animals. However, dexamethasone reduced IL-2R expression on activated hepatic $\mathrm{T}$ cells isolated from control rats, but failed to attenuate IL- $2 \mathrm{R}$ upregulation in ANIT treated rats (fig 7). 


\section{DISCUSSION}

Glucocorticoids have a wide range of biological effects with potent anti-inflammatory functions, in part through their ability to inhibit the expression of inflammatory cytokines such as IL-2 and IFN- $\gamma^{25}$ Thl cytokines are typically proinflammatory in nature and play a central role in cell mediated immunity. An augmented and overzealous Thl response may be detrimental to tissues, and is known to play an important role in the pathogenesis of several autoimmune diseases. ${ }^{26-28}$ Despite a significant rise in the serum glucocorticoid levels in rats with experimental cholangitis, we observed a predominant hepatic Thl cytokine profile, which we have previously demonstrated to be due to enhanced IFN- $\gamma$ production by hepatic $\mathrm{CD} 4^{+} \mathrm{T}$ lymphocytes. ${ }^{5}$ Our data suggest that hepatic T lymphocytes in ANIT treated rats are insensitive to the immunomodulatory actions of endogenous glucocorticoids. This insensitivity of hepatic $\mathrm{T}$ lymphocytes to glucocorticoid mediated suppressive effects would be expected to directly aggravate hepatic inflammation. Interestingly, clinical studies have shown that corticosteroid treatment in patients with chronic cholangitic diseases such as PSC and PBC, either alone or in conjunction with other drugs such as ursodeoxycholic acid, does not appear to be effective. ${ }^{14-16}$

Resistance of T lymphocytes to glucocorticoid effects has been documented in a number of chronic inflammatory diseases including asthma and IBD. ${ }^{13} 29$ Steroid resistance in these conditions has been attributed, at least in part, to GR defects. In patients with IBD, GR numbers in peripheral blood mononuclear cells (PBMCs) are significantly lower in steroid non-responsive patients compared with steroid responders and healthy subjects. ${ }^{10}$ Moreover, IBD patients have been shown to have elevated serum cortisol levels with lower levels of serum DHEA compared with control subjects, ${ }^{30}$ a similar finding to our observation in rats with experimental cholangitis. Our results demonstrate that hepatic $\mathrm{T}$ lymphocytes isolated from ANIT treated rats have decreased GR expression compared with controls, and we suggest that this directly contributes to glucocorticoid insensitivity of these cells. GR downregulation does not appear to be a consequence of elevated circulating glucocorticoids as similar GR expression was documented in T lymphocytes isolated from the spleen and peripheral blood of control and ANIT treated rats.

GR expression may vary in different tissues or cell populations; therefore, we ensured that similar cell populations were being compared. Fluorescence activated cell sorter (FACS) analysis of isolated liver derived lymphocytes demonstrated that GR downregulation in hepatic T cells from ANIT treated rats was not due to differences in cell populations between control and ANIT treated rats, as both control and ANIT groups showed comparable cell types and distribution. Liver derived lymphocytes from both control and ANIT fed rats consist mainly of $\mathrm{T}$ and NK cells with low or negligible numbers of monocytes/macrophages and B cells. A similar observation was noted in liver derived lymphocytes from patients with PBC and PSC. ${ }^{31}$ Interestingly, our double staining study showed that the great majority of NK positive cells in liver derived lymphocytes isolated from control and ANIT treated animals were also CD8 positive, similar to the observations of others. ${ }^{32}$ Although NK cells typically do not express CD3, they have been shown to express CD4 or CD8 surface markers. In our study, liver derived lymphocytes in control and ANIT treated rats consisted of a much lower percentage of $\mathrm{NK} / \mathrm{CD}^{+}$cells than NK/CD8 ${ }^{+}$cells. Although we did not measure the relative percentage of NK positive cells in the peripheral blood of ANIT fed rats, we did observe a low percentage of circulating NK positive cells in control rats. Accumulation of NK cells in the liver may play a direct or indirect role in liver immune dysfunction; however, their role in the development of chronic cholangitis is unknown.

Reduced cellular sensitivity to GC mediated suppression may be associated with lowered GR number and/or affinity.
However, reduced GR numbers may not always translate into reduced GR function. Schlaghecke et al demonstrated that GR function (that is, GC inhibition of lymphocyte proliferation and cytokine release) is not compromised despite reduced GR number in PBMC of patients with rheumatoid arthritis. ${ }^{33}$ To determine whether hepatic T cell GR downregulation in rats with chronic cholangitis was associated with reduced GR function, hepatic T cell ConA stimulated IL-2R expression was analysed in the presence or absence of dexamethasone using FACS analysis. GCs have been shown to inhibit cytokine receptor (for example, IL-2R) expression on activated $\mathrm{T}$ cells. ${ }^{23}$ In our study, dexamethasone attenuated ConA induced IL-2R expression on hepatic T cells of control rats but failed to inhibit ConA stimulated IL-2R expression on hepatic T cells from ANIT treated rats. Therefore, our results suggest that in rats with experimental chronic cholangitis, hepatic T cell GR downregulation appears to impair GR function (that is, inhibition of IL-2R expression).

The mechanism for GR downregulation in hepatic $\mathrm{T}$ lymphocytes of ANIT fed rats is not known. Various factors such as ligand induced downregulation, inflammatory cytokines, and cellular activation may influence GR expression. Glucocorticoids are known to regulate their own receptor expression at various levels (reviewed by Oakley and Cidlowski ${ }^{34}$ ). Given that GR downregulation in ANIT treated rats is tissue specific, and not a generalised effect in response to elevated circulating glucocorticoid levels, it seems unlikely that hepatic T lymphocyte GR downregulation in ANIT treated rats is simply due to glucocorticoid mediated autoregulation of receptor expression.

In recent years, a number of cytokines have been shown to modulate GR number and/or affinity, thereby contributing to glucocorticoid resistance. Kam et al demonstrated that incubation of human PBMCs with both IL-2 and IL-4 together resulted in a decrease in GR affinity without a change in GR number. ${ }^{35}$ However, IL-2 and IL-4 are unlikely to play a significant role in decreased hepatic T cell GR expression in ANIT fed rats for two reasons. Firstly, these cytokines do not appear to cause a reduction in GR number. ${ }^{35}$ Secondly, hepatic IL-4 levels in ANIT treated rats did not differ from those observed in control rats. However, other cytokines may play a role in modulating GR expression in hepatic $\mathrm{T}$ cells of rats with chronic cholangitis.

In addition, stimulation of immune cells with phorbol esters and the cytokine tumour necrosis factor $\alpha$ (TNF- $\alpha$ ) have been shown to modulate cellular GR number. ${ }^{36-38}$ However, we found that incubation of splenic T cells with either TNF- $\alpha$ or phorbol-12- myristate 13-acetate for up to 48 hours did not decrease cellular GR numbers (Tjandra K, unpublished data).

In summary, hepatic T lymphocyte resistance to elevated circulating glucocorticoids occurs in rats with experimental cholangitis. The mechanism for steroid resistance in this model may be attributed, at least in part, to downregulation of GR in hepatic T lymphocytes. Hepatic T cell GR downregulation in ANIT induced experimental cholangitis is tissue specific and appears to be independent of circulating glucocorticoid levels. Currently, the mechanism underlying GR downregulation in hepatic T lymphocytes during experimental cholangitis is unknown but is of obvious potential clinical relevance in treating these diseases. Our findings may explain, at least in part, why patients with chronic cholangitis do not respond clinically to glucocorticoid therapy.

\section{ACKNOWLEDGEMENTS}

This study was supported by a grant from the Canadian Liver Foundation. MGS is an Alberta Heritage Foundation for Medical Research Scholar.

\section{Authors' affiliations}

K Tjandra, T Le, M G Swain, Gastrointestinal Research Group, University of Calgary, Calgary, Alberta, Canada T2N 1N4 


\section{REFERENCES}

1 Wiesner RH, Grambsch PM, Dickson ER, et al. Primary sclerosing cholangitis: natural history, prognostic factors and survival analysis. Hepatology 1989;10:430-6.

2 Farrant JM, Hayllar KM, Wilkinson ML, et al. Natural history and prognostic variables in primary sclerosing cholangitis. Gastroenterology 1991:100:1710-17.

3 Porayko MK, LaRusso NF, Wiesner RH. Primary sclerosing cholangitis: a progressive disease? Semin Liver Dis 1991;11:18-25.

4 Berg PA, Klein R, Rocken M. Cytokines in primary biliary cirrhosis. Semin Liver Dis 1997; 17: 115-23.

5 Tiandra K, Sharkey KA, Swain MG. Progressive development of a Th 1 -type hepatic cytokine profile in rats with experimental cholangitis. Hepatology 2000;31:280-90.

6 Ramierz F, Fowell DJ, Puklavec M, et al. Glucocorticoids promote a TH2 cytokine response by CD4+ T cells in vitro. J Immunol 1996;156:240612 .

7 Daynes RA, Araneo BA, Dowell TA, et al. Regulation of murine lymphokine production in vivo. III. The lymphoid tissue microenvironment exerts regulatory influences over T helper cell function. J Exp Med 1990;171:979-96.

8 Daynes RA, Dudley DJ, Araneo BA. Regulation of murine lymphokine production in vivo. II. Dehydroepiandrosterone is a natural enhancer of interleukin 2 synthesis by helper T cells. Eur J Immunol interleukin 2 synthesis
$1990 \cdot 20 \cdot 793-802$

9 Swain MG, Maric M. Prevention of immune-mediated arthritis in cholestatic rats: involvement of endogenous glucocorticoids. Gastroenterology 1994;107: 1469-74.

10 Madretsma GS, van Dijk APM, Wilson JHP, et al. Corticosteroid resistance in colitis is characterized by a low glucocorticoid receptor content of mononuclear cells. Gut 1995:37(suppl 2):A178.

11 Rogler G, Meinel A, Lingauer A, et al. Glucocorticoid receptors are down-regulated in inflamed colonic mucosa but not in peripheral blood mononuclear cells from patients with inflammatory bowel disease. Eur Clin Invest 1999;29:330-6.

12 Adcock IM, Lane SJ, Brown CR, et al. Differences in binding of glucocorticoid receptor to DNA in steroid- resistant asthma. J Immunol 1995:154:3500-5.

13 Syed $\mathbf{F}$, Bingham $B$, Johnson $M$, et al. The CD4+ T lymphocyte is a site of steroid resistance in asthma. QJ Med 1998:91:567-72.

14 Mitchison HC, Bassendine MF, Malcolm AJ, et al. A pilot, double-blind, controlled 1 -year trial of prednisolone treatment in primary biliary cirrhosis: hepatic improvement but greater bone loss. Hepatology 1989; 10:420-9.

15 van Hoogstraten HJ, Vleggaar FP, Boland GJ, et al. Budesonide or prednisone in combination with ursodeoxycholic acid in primary sclerosing cholangitis: a randomized double-blind pilot study. Belgian-Dutch PSC Study Group. Am J Gastroenterol 2000;95:2015Belgian

16 Angulo $\mathbf{P}$, Jorgensen RA, Keach JC, et al. Oral budesonide in the treatment of patients with primary biliary cirrhosis with a suboptimal response to ursodeoxycholic acid. Hepatology 2000;31:318-23.

17 Sarlis NJ, Chowdrey HS, Stephanou A, et al. Chronic activation of the hypothalamo- pituitary-adrenal axis and loss of circadian rhythm during adjuvant-induced arthritis in the rat. Endocrinology 1992;130:1775-9.

18 Swain MG, Le T, Tigley AW, et al. Hypothalamic nitric oxide synthase is depressed in cholestatic rats. Am J Physiol 1997;272:G1034-40.

19 Swain MG, Appleyard C, Wallace J, et al. Endogenous glucocorticoids released during acute toxic liver injury enhance hepatic IL- 10 synthesis and release. Am J Physiol 1999;276:G199-205.
20 Miller AH, Spencer RL, Pearce BD, et al. Glucocorticoid receptors are differentially expressed in the cells and tissues of the immune system. Cell Immunol 1998:186:45-54

21 Lai KN, Leung J, CLai FM. Soluble interleukin 2 receptor release, interleukin 2 production, and interleukin 2 receptor expression in activated T-lymphocytes in vitro. Pathology 1991;23:224-8.

22 Berguer R, Dalton M, Ferrick D. Adrenocortical response and regional T-lymphocyte activation patterns following minimally invasive surgery in a rat model. Surg Endosc 1998;12:236-40.

23 Xia M, Gasser J, Feige U. Dexamethasone enhances CTLA-4 expression during T cell activation. Cell Mol Life Sci 1999;55:1649-56.

24 Hata K, Zhang XR, Iwatsuki S, et al. Isolation, phenotyping, and functional analysis of lymphocytes from human liver. Clin Immunol Immunopathol 1990:56:401-19.

25 Franchimont $\mathbf{D}$, Louis $E$, Dewe W, et al. Effects of dexamethasone on the profile of cytokine secretion in human whole blood cell cultures. Regul Pept 1998;73:59-65.

26 Quayle AJ, Chomarat P, Miossec P, et al. Rheumatoid inflammatory T-cell clones express mostly Th1 but also Th2 and mixed (Th0-like) cytokine patterns. Scand J Immunol 1993;38:75-82.

27 Benvenuto R, Paroli M, Buttinelli C, et al. Tumour necrosis factor-alpha synthesis by cerebrospinal-fluid-derived T cell clones from patients with multiple sclerosis. Clin Exp Immunol 1991;84:97-102.

28 Selmai K, Raine CS, Cannella B, et al. Identification of lymphotoxin and tumor necrosis factor in multiple sclerosis lesions. J Clin Invest 1991:87:949-54.

29 Hearing SD, Norman M, Probert CS, et al. Predicting therapeutic outcome in severe ulcerative colitis by measuring in vitro steroid sensitivity of proliferating peripheral blood lymphocytes. Gut 1999:45:382-8.

30 Straub RH, Vogl D, Gross V, et al. Association of humoral markers of inflammation and dehydroepiandrosterone sulfate or cortisol serum levels in patients with chronic inflammatory bowel disease. Am J Gastroenterol 1998;93:2197-202.

31 Hata K, Van Thiel DH, Herberman RB, et al. Natural killer activity of human liver- derived lymphocytes in various liver diseases. Hepatology $1991 ; 14: 495-503$

32 Seaman WE. Natural killer cells and natural killer T cells. Arthritis Rheum 2000;43:1204-17

33 Schlaghecke R, Beuscher D, Kornely E, et al. Effects of glucocorticoids in rheumatoid arthritis. Diminished glucocorticoid receptors do not result in glucocorticoid resistance. Arthritis Rheum 1994;37:1127-31.

34 Oakley RH, Cidlowski JA. Homologous down regulation of the glucocorticoid receptor: the molecular machinery. Crit Rev Eukaryot Gene Expr 1993;3:63-88

35 Kam JC, Szefler SJ, Surs W, et al. Combination IL-2 and IL-4 reduces glucocorticoid receptor-binding affinity and $T$ cell response to glucocorticoids. J Immunol 1993;151:3460-6.

36 Crabtree GR, Munck A, Smith KA. Glucocorticoids and lymphocytes. I. Increased glucocorticoid receptor levels in antigen-stimulated lymphocytes. J Immunol 1980;124:2430-5.

37 Smith KA, Crabtree GR, Kennedy SJ, et al. Glucocorticoid receptors and glucocorticoid sensitivity of mitogen stimulated and unstimulated human lymphocytes. Nature 1977:267:523-6.

38 Franchimont D, Martens H, Hagelstein MT, et al. Tumor necrosis factor alpha decreases, and interleukin-10 increases, the sensitivity of human monocytes to dexamethasone: potential regulation of the glucocorticoid receptor. J Clin Endocrinol Metab 1999;84:2834-9. 